\title{
Capillary refilling time in newborn babies: normal values
}

\author{
Krzysztof S Strozik, Clarissa H Pieper, Jacques Roller
}

\begin{abstract}
Aim-To assess the normal values of capillary refilling time (CRT) in healthy newborn babies; to assess the effect of different nursery containers (incubator, radiant warmer, crib), phototherapy, birthweight, gestational age, size for gestational age and sex on CRT; to compare CRT at different body sites as well as to assess the variation between observers.

Methods-Healthy neonates $(n=469)$ of different gestational ages and different sizes for gestational age, were studied 1 to 7 days after birth. CRT was measured in four of the most suitable sites-namely, midpoints of the sternum and the forehead, the palm of the hand and the plantar surface of the heel (defined as chest, head, palm and heel, respectively). The applied pressing time was 5 seconds. CRT was measured with a manual stopwatch.
\end{abstract}

Results-Only the chest and the head distribution curves followed the Gaussian curve. The mean values and standard deviation of CRT in all tested nursery containers, including phototherapy for the chest, ranged from $1.82(0.34)$ seconds to $2.01(0.423)$ seconds, and for the head from $1.59(0.36)$ seconds to $1.83(0.31)$ seconds. The mean value of chest CRT was always longer than the head CRT for all parameters. Significant differences were found between different nursery containers, receivers, and non-receivers of phototherapy and between observers. No difference was found between sex, birthweight, gestational age and size for gestational age.

Conclusions-The upper limit of normal for neonatal CRT was 3 seconds. Nursery containers, phototherapy, and observers produced significantly different results, but the differences were not clinically important. CRT values of the midpoints of the sternum and the forehead are the most consistent.

(Arch Dis Child 1997;76:F193-F196)

Keywords: capillary refilling time; nursery containers; phototherapy

Capillary refilling time (CRT) is a widely accepted method of assessing cardiac output and peripheral vascular resistance..$^{1-3}$ Surprisingly, its introduction into clinical practice was not preceded by detailed research. ${ }^{4}$ Only a few trials were conducted to estimate normal values and clinical applications of CRT, and the conclusions drawn were inconsistent. ${ }^{5-10}$ In three published studies, ${ }^{6}{ }^{10}$ the normal CRT in children was established but this study is the first to assess CRT in healthy neonates as well as to assess the impact of a range of variables on CRT.

\section{Methods}

The study was performed in Tygerberg Hospital in two neonatal nurseries (one each for preterm and term neonates) between January and September 1994. Only healthy neonates 1 to 7 days old, with an axillary body temperature documented and observed to be between 36$37^{\circ} \mathrm{C}$ before and after the test, and who were clinically healthy with normal observations for the preceding 24 hours, were included. For at least 2 hours before the survey the baby was kept in one of the following: incubator; radiant warmer; crib; or under phototherapy lights.

Neonates who presented with cold extremities immediately before the measurement, and those with any clinically definable abnormality, were excluded.

Informed consent was obtained from a parent of each infant included in the study.

For the purpose of this study, capillary refilling time was measured in four body areas: midpoint of the sternum; midpoint of the forehead; palm of the hand; and plantar surface of the heel (in this study - chest, head, palm and heel, respectively).

Measurements were performed by applying minimal pressure (defined as the pressure which produced blanching) with the examiner's thumb. Applied pressing time was 5 seconds. CRT was measured in every patient by the simultaneous activation of the stopwatch and the application of minimal pressure until the skin blanched. At the end of 5 seconds the pressure was released. When skin colour returned to baseline the stopwatch was inactivated. The patients were assessed lying supine in an incubator, radiant warmer, or crib. The CRT on each baby was then measured starting at the head, then the chest, followed by the palm and the heel. Each newborn was assessed once only. If for any reasons the measurement had to be repeated, a period of 30 seconds was allowed before the next attempt. Incubators (Isolette) and radiant warmers (AMA) with servo controlled temperature were used.

All babies were examined in well lit rooms with a controlled, stable, ambient temperature of $23^{\circ} \mathrm{C} \pm 1^{\circ} \mathrm{C}$. The incubators and radiant warmers were servo controlled to maintain the temperature at $36.6^{\circ} \mathrm{C}$. All preterms weighing under $1800 \mathrm{~g}$ were nursed in incubators. Estimation of the size for gestational age (AGA, LGA, SGA) was based on standard infant 
Table 1 Analysis of capillary refilling time results of chest and head

\begin{tabular}{|c|c|c|c|c|}
\hline Variable & $\begin{array}{l}\text { Mean (SD) chest } \\
\text { (seconds) }\end{array}$ & P value & $\begin{array}{l}\text { Mean (SD) head } \\
\text { (seconds) }\end{array}$ & Pvalue \\
\hline Phototherapy: & & NS & & 0.02 \\
\hline Non-receivers $(n=384)$ & $1.89(0.37)$ & & $1.75(0.38)$ & \\
\hline Receivers $(n=85)$ & $1.91(0.43)$ & & $1.65(0.42)$ & \\
\hline Nursing containers: & & 0.0075 & & 0.0001 \\
\hline Incubator $(n=81)$ & $2.01(0.42)$ & & $1.62(0.41)$ & \\
\hline Radiant warmer $(n=78)$ & $1.83(0.45)$ & & $1.59(0.36)$ & \\
\hline Crib $(n=310)$ & $1.88(0.35)$ & & $1.79(0.35)$ & \\
\hline Observer: & & 0.002 & & NS \\
\hline KSS & $1.95(0.42)$ & & $1.72(0.41)$ & \\
\hline $\mathrm{JR}$ & $1.84(0.31)$ & & $1.74(0.30)$ & \\
\hline Sex: & & NS & & NS \\
\hline Boys & $1.89(0.38)$ & & $1.71(0.34)$ & \\
\hline Girls & $1.90(0.38)$ & & $1.74(0.40)$ & \\
\hline Birthweight: & & NS & & NS \\
\hline$<2500 \mathrm{~g}(\mathrm{n}=169)$ & $2.00(0.35)$ & & $1.69(0.34)$ & \\
\hline$>2500 \mathrm{~g}(\mathrm{n}=300)$ & $1.84(0.41)$ & & $1.76(0.41)$ & \\
\hline Gestation: & & NS & & NS \\
\hline$<37$ weeks $(\mathrm{n}=203)$ & $1.98(0.41)$ & & $1.72(0.41)$ & \\
\hline$>38$ weeks $(n=266)$ & $1.82(0.34)$ & & $1.73(0.34)$ & \\
\hline Size for gestation age: & & NS & & NS \\
\hline $\mathrm{AGA}(\mathrm{n}=317)$ & $1.90(0.39)$ & & $1.74(0.37)$ & \\
\hline SGA $(n=95)$ & $1.93(0.38)$ & & $1.60(0.38)$ & \\
\hline LGA $(n=57)$ & $1.84(0.34)$ & & $1.83(0.31)$ & \\
\hline Total $(n=469)$ & $1.90(0.38)$ & & $1.73(0.37)$ & \\
\hline
\end{tabular}

weight at birth charts used in Tygerberg Hospital.

All the measurements were carried out by two independent observers (KS and JR), but every baby was assessed by only one observer. The time was recorded by the same stopwatch and measured in tenths of a second.

This is a descriptive study of all available babies during the study period. The data were analysed using multivariate analysis of variance, using the SATS computer program as well as Statgraphics on a standard PC. Levels of significance reported in table 1 were obtained as those applicable for $\mathrm{F}$ statistics generated by the statistical program.

\section{Results}

The gestational age of the 469 neonates examined varied between 28 and 42 weeks. The characteristics of the patients are listed in table 1. Of the 85 who received phototherapy, 37 were nursed in closed incubators, 46 in cribs, and two under radiant warmers.

The results of all the investigations on chest, head, palm and heel showed that the distribution curves of the test for the chest and the head followed a Gaussian curve (figs 1 and 2). The results for the palm and heel showed a diffuse scatter of the values (figs 3 and 4). Therefore, only the chest and head data were analysed in detail.

The mean and the upper limit of normal CRTs in the total group of 469 babies were: chest 1.9 and 2.66 seconds; head 1.73 and 2.47

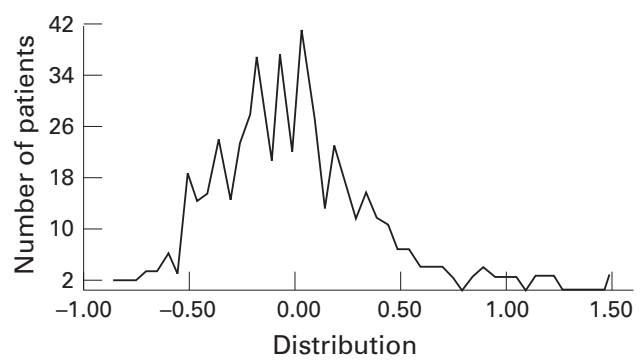

Figure 1 Distribution curves of CRT values for the chest. seconds. The upper limit of normal chest and head CRTs in different subgroups-receivers or non-receivers of phototherapy, nursery containers, sex, gestational age, size for gestational age, and observers-was never more than 3 seconds (fig 5).

Statistical analysis of the results showed that sex of the babies, birthweight, gestational age and size for gestational age did not influence CRT. The CRT during phototherapy, in different nursery containers (crib, incubator, or radiant warmer) and measured by different investigators, varied significantly (table 1 ).

The mean and the upper limit of normal CRT in the palm and heel were 1.90, 2.82, and 2.14 and 3.22 seconds, respectively. The further analysis of the palm and heel data was abandoned because of the diffuse scatter of values.

\section{Discussion}

Prolonged CRT is one of the few signs of circulatory compromise in newborn babies that can be measured without expensive equipment. Although this method was first introduced in 1947, and normal values were reported in 1980, many aspects of CRT have still not been investigated. ${ }^{11}{ }^{12}$ The clinical value of this test was recently questioned. ${ }^{4910}$ The difficulty in establishing the real value of CRT as a useful clinical tool lies not only in the paucity of clinical trials, but also in the different methods applied in existing studies, such as different pressing times and sites of testing, small numbers, and wide variations among participants. $^{5-7} 910$ Studies in homogeneous groups of neonates have not been reported.

Neonatal wards with stable room temperatures are ideal for CRT application, because of the influence of ambient temperature on CRT. ${ }^{70}$ Decreased local skin temperature with diminished local skin blood flow can prolong CRT. $^{713}$

The upper limit of the CRT in neonates was found to be 3 seconds. This value is applicable to all neonates, irrespective of sex, gestation, weight, size for gestational age, nursery containers and phototherapy. This contrasts with values of 1.9 seconds found in a group of children aged between 2 weeks and 12 years. ${ }^{67}$ An upper limit of 2 seconds for the finger and 3 seconds for the heel was found in a small study by Corelick. ${ }^{10}$ Comparing the results, one should keep in mind the big difference in methodology (different sites, pressing times and age groups) The CRT was shorter for newborns nursed in radiant warmers or receiv-

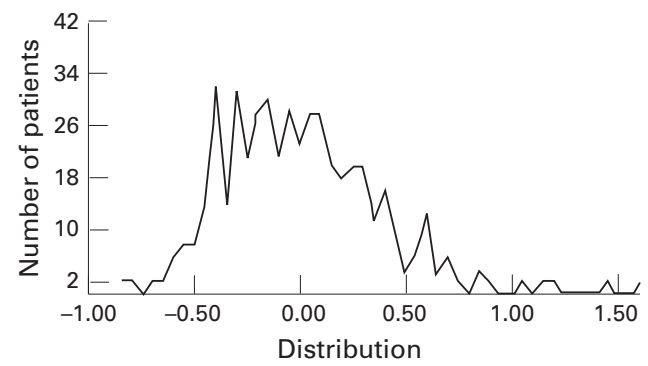

Figure 2 Distribution curves of CRT values for the head. 


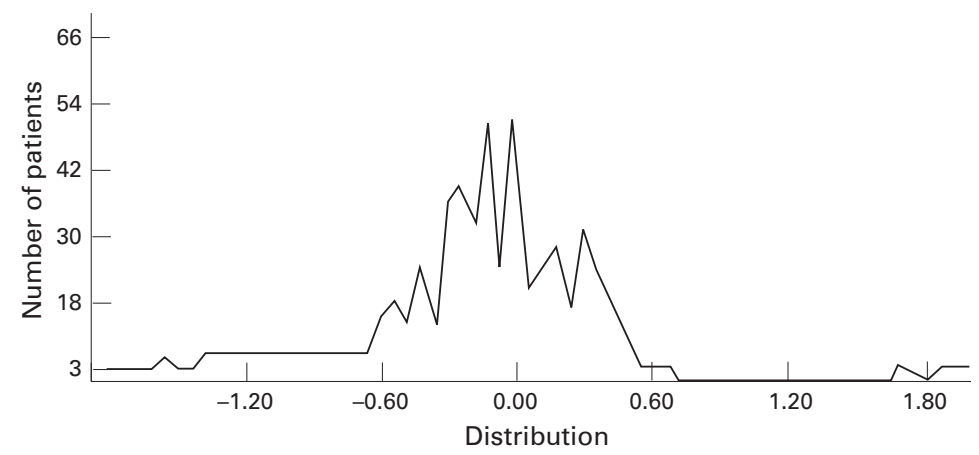

Figure 3 Distribution curves of CRT values for the palm.

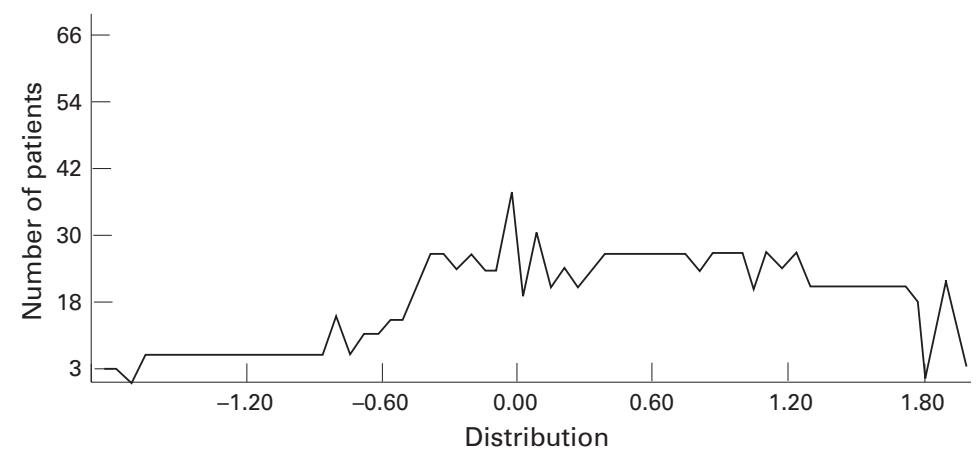

Figure 4 Distribution curves of CRT values for the heel.

ing phototherapy and for those who were nursed in incubators. This can be explained by the influence of the higher ambient temperature and possibly phototherapy that may have increased local skin blood flow, because this directly influences CRT. ${ }^{10}$ Although the CRT of neonates in radiant warmers, incubators, and under phototherapy was significantly shorter, it does not seem to be clinically relevant. The difference of 0.2 seconds for forehead, and 0.18 seconds for chest between the longest and the shortest mean value cannot be detected using ordinary clinical methods. We believe that a difference of at least 1 second is needed for this simple bedside test to be clinically measurable ( 3 vs 4 seconds).

Other variables such as sex did not influence CRT. In previous reports sex was one of the determining factors, but this was in an adult population. ${ }^{6}$ Surprisingly, birthweight, gestational age, and nutritional status determined as size for gestational age (AGA, SGA, LGA) did not affect CRT. Factors such as skin thickness, blood pressure, and percentage of total body water, which can influence CRT, differ in preterm and term, AGA, SGA, or LGA babies. In fact, no study has determined actual skin blood flow which directly affects CRT in these groups of neonates. The fact that most preterm newborns are nursed in incubators or radiant warmers, and term newborns in cribs, could also have influenced our findings.

We tested CRT at four sites: midpoints of the sternum and forehead, palm of the hand, and plantar surface of the heel. In previous studies the distal phalanx of middle or index finger, the nailbed of the thumb, chest wall, forehead, lip mucosa or the heel were tested..$^{5-7} 9{ }^{10}$ We found that the midpoints of the sternum and forehead

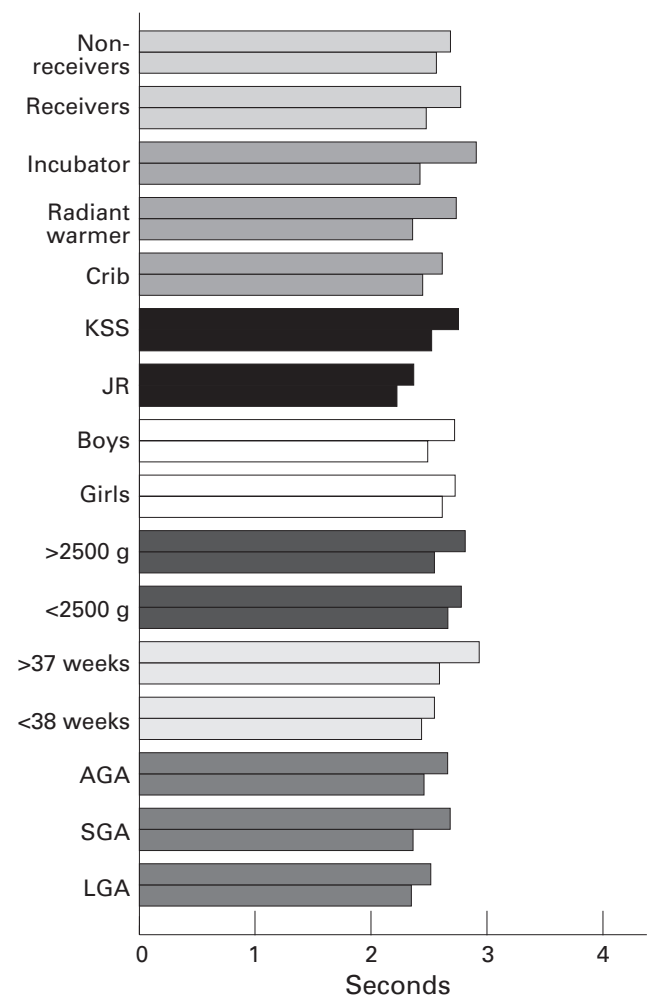

Upper Bar $=$ Chest upper limit of normal capillary filling time

Lower Bar = Head upper limit of normal capillary filling time

\begin{tabular}{|l}
$\square$ Phototherapy \\
Nursing containers \\
Observer \\
$\square$ Sex \\
Birthweight \\
$\square$ Gestation \\
$\square$ Size for gestational age
\end{tabular}

Figure 5 Upper limit of normal CRT in different groups of patients.

were the most practical and reliable for testing. The distribution curves of the tests of the chest and forehead followed a Gaussian distribution curve. The baby's movements do not interfere with measurements in these areas. The results of the palm and heel showed a diffuse scatter of values, which can be attributed to difficulties in measurement.

Testing of CRT in finger pulp or nailbeds was impractical, because of the small size of these areas especially in preterm infants. The values of CRT obtained on the head were significantly shorter than the chest values. The explanation for this is difficult and may be related to differences in local skin blood flow.

Because every baby was assessed by only one observer, it was impossible to estimate interobserver variability. But there were sufficient numbers of participants to compare the variability of data obtained by the two observers. Although the difference for the chest was significant, from a clinical point of view this was too small to be of any clinical importance.

We found the CRT to have absolute values of normality. It is an easy, fast, and reliable test if done in the right areas of the body. More studies need to be done to establish its potential 
clinical applications in newborn babies, especially as a tool to differentiate between healthy and sick newborns.

We thank Miss S Engelbrecht for typing the manuscript and the Department of Statistics, University of Stellenbosch, for consultation.

1 Robson AM. Parenteral fluid therapy. In: Behrman RE, Vaughan VC, eds. Nelson Textbook of Pediatrics. 13th edn. Philadelphia: WB Saunders \& Co, 1987:195.

2 White R. In: Baldwin CA, ed. Handbook of Pediatric Emergencies. 1st edn. Boston: Little, Brown Co, 1989: 42

3 Levy HB, Sheldon SH, Sulayman RF Diagnosis and Management of the Hospitalised Child. New York: Raven Press, 1984:517.

4 Baraff L J. Capillary refill: Is it a useful clinical sign? Pediatrics 1993;92:723-4.

5 Saavedra JM, Harris GD, Li S, Finberg L. Capillary refilling (skin turgor) in the assessment of dehydration. Am $\mathcal{F}$ Dis
6 Schringer DL, Baraff LJ. Determination and comparison of normal capillary refill between healthy children and adults. Pediatr Res 1988;23:236A.

7 Schringer DL. Baraff LJ. Defining normal capillary refill: variation with age, sex and temperature. Ann Emerg Med 1988;17:932-5.

8 Mackenzie A, Barnes G, Shann F. Clinical signs of dehydration in children. Lancet 1989;ii:605-7.

9 Schringer DL, Baraff LJ. Capillary refill - is it a useful predictor of hypovolemic states? Ann Emerg Med 1991;20:601-5.

10 Gorelick MH, Shaw KN, Baker MD. Effect of ambient temperature on capillary refill in normal children. Pediatrics 1993;92:699-702.

11 Beecher HK, Simeone FA, Burnett $\mathrm{CH}$, et al. The internal state of the severely wounded man on entry to the most forward hospital. Surgery 1947;22:627-31

12 Champion HR, Sacco WJ, Hannan DS, et al. Assessment of injury severity: the Triangle Index. Crit Care Med 1980;8:201-8.

13 Johnson JM, Brengelmann GL, Hales JRS, et al. Regulation of cutaneous circulation. Fed Proc 1986;45:2841-50. 